\title{
Two emission line objects with $z>0.2$ in the optical filament apparently connecting the Seyfert galaxy NGC 7603 to its companion
}

\author{
M. López-Corredoira ${ }^{1,2}$ and C. M. Gutiérrez ${ }^{1}$ \\ 1 Instituto de Astrofísica de Canarias, 38200 La Laguna, Tenerife, Spain \\ 2 Astronomisches Institut der Universität Basel, Venusstrasse 7, 4102 Binningen, Switzerland \\ Received 24 January 2002 / Accepted 26 March 2002

\begin{abstract}
We present new spectroscopic observations of an old case of anomalous redshift - NGC 7603 and its companion. The redshifts of the two galaxies which are apparently connected by a luminous filament are $z=0.029$ and $z=0.057$ respectively. We show that in the luminous filament there are two compact emission line objects with $z=0.243$ and $z=0.391$. They lie exactly on the line traced by the filament connecting the galaxies. As far as we are aware, this is the most impressive case of a system of anomalous redshifts discovered so far.
\end{abstract}

Key words. galaxies: individual: NGC 7603 - quasars: general - galaxies: statistics - galaxies: peculiar distance scale

\section{Introduction}

Thirty years ago it was shown that NGC 7603 (Mrk 530, Arp 92) is a remarkable example of an anomalous redshift association (Arp 1971). NGC 7603 is a Seyfert 1 galaxy with strong spectral variability (Kollatschny et al. 2000). A smaller galaxy (NGC 7603B, denoted by object 1 in Fig. 1) lies at the end of a filament which apparently connects both galaxies. The galaxies have redshifts corresponding to $8700 \mathrm{~km} \mathrm{~s}^{-1}$ and $17000 \mathrm{~km} \mathrm{~s}^{-1}$ respectively.

Sharp (1986) suggested that the absence of emission lines in the smaller galaxy argued against a possible interaction between NGC 7603 and the smaller companion (NGC 7603B $\equiv$ object 1). However, the non-detection of emission lines is not proof against the existence of a physical connection. In interactions and ejections with a larger galaxy, the gas is often stripped out of a stellar system; so the lack of emission lines could be taken as an indication of interaction rather than non interaction. It might also be that NGC 7603B, although roughly at the same distance, is not close enough to NGC 7603 to have tidal effects and star formation.

NGC 7603 and other examples, in which two galaxies with different redshifts are apparently connected by a filament, have been considered fortuitous by most researchers. However, we decided that a deep analysis of this system should be carried out to try to give an answer to the controversy. We considered it important to determine the redshifts of the two observed knots

Send offprint requests to: M. López-Corredoira,

e-mail: martinlc@astro.unibas.ch
(Arp 1971) (objects 2 and 3) in the filament. For some time after the discovery of the system, attempts were made to obtain spectra of the objects in the bridge, but because of limitations of the equipment available in the 1970s, none was successful (M. Burbidge, private comm.). In this paper we report observations in which we were succesful in obtaining spectra of not two but four objects connected by the filament. In Sect. 3, we discuss the spectra of objects 2 and 3 .

\section{Observations}

Table 1 summarizes the observations ${ }^{1}$ used in this paper. The image in $R$-band (Fig. 1a) shows NGC 7603 with 3 companions: NGC 7603B (object 1), object 2 and object 3. We took long-slit spectra of the southern part of NGC 7603B, and objects 2 and 3; all the spectra were obtained within the same 5 arcsec-width slit indicated by a dashed line in Fig. $1 \mathrm{~b}$.

\section{Discrepant redshifts}

Figures 1a,b show clearly the filament between NGC 7603 and NGC 7603B (object 1). A knot (object 2) is perfectly centered in the line of the filament and positioned where the filament connects to NGC 7603B. The other knot (object 3) is also

\footnotetext{
${ }^{1}$ Based on observations made with the Nordic Optical Telescope, operated on the island of La Palma jointly by Denmark, Finland, Iceland, Norway, and Sweden, in the Spanish Observatorio del Roque de los Muchachos of the Instituto de Astrofísica de Canarias.
} 
Table 1. Source of NGC 7603 data used in this paper: $R$-band image as shown in Fig. 1, and a long-slit spectrum along the dashed lines marked in Fig. 1b whose extracted spectra are shown in Fig. 2.

\begin{tabular}{|c|c|c|}
\hline - & $R$-band image & slit-spectrum 4000-7000 A \\
\hline Telescope & NOT-2.6 m. (La Palma-Spain) & NOT-2.6 m. (La Palma-Spain) \\
\hline Instrument & ALFOSC & ALFOSC/grism 4, aperture: $5^{\prime \prime}$ \\
\hline Resolution & $0.188^{\prime \prime} /$ pixel & $0.188^{\prime \prime} /$ pixel; $2.96 \AA$ A $/$ pixel \\
\hline Date & 2000, June 13th & 2001, August 12th \\
\hline Exposure time/Moon & 900 s./dark & 14225 s./grey-dark \\
\hline
\end{tabular}

Table 2. Characteristics of the observed objects.

\begin{tabular}{cccccc}
\hline \hline object & Spectral lines & structure & Eq. coordinates (J2000) & magnitude & redshift (heliocentric) \\
\hline 1 (NGC 7603B) & absorption & extended & $\alpha=23^{\mathrm{h}} 19^{\mathrm{m}} 00.1^{\mathrm{s}}, \delta=+0^{\circ} 14^{\prime} 7^{\prime \prime}$ & $m_{B}=16.8^{1}$ & $0.058 \pm 0.002$ \\
2 & emission & point-like & $\alpha=23^{\mathrm{h}} 18^{\mathrm{m}} 59.4^{\mathrm{s}}, \delta=+0^{\circ} 14^{\prime} 4^{\prime \prime}$ & $m_{R}=21.8 \pm 0.2$ & $0.243 \pm 0.001$ \\
3 & emission & point-like & $\alpha=23^{\mathrm{h}} 18^{\mathrm{m}} 57.7^{\mathrm{s}}, \delta=+0^{\circ} 14^{\prime} 2^{\prime \prime}$ & $m_{R}=21.4 \pm 0.2$ & $0.391 \pm 0.001$ \\
Filament & absorption & extended & from NGC 7603 to NGC 7603B & $\sim 23.5 / \mathrm{arcsec}^{2}$ & $0.030 \pm 0.001$ \\
\hline
\end{tabular}

${ }^{1}$ Sharp (1986).

perfectly centered to within 1 arcsec in the filament, and is positioned where the filament connects with NGC 7603. There is also a second filament which sweeps around from the main galaxy through the position of the companion NGC 7603B (Arp 1971, 1975). The halo of the common system extends over the northern area of NGC 7603B, that is at the left side of the center of NGC 7603 in Fig. 1a. However, the halo does not extend on the right position in the same way.

We have determined the redshift of the objects 1 (NGC 7603B) to 3. Table 2 summarizes the information about these objects. Figure $2 \mathrm{a}$ shows the spectra of the objects 2 and 3, which are emission line sources with redshifts 0.243 and 0.391 respectively. $\mathrm{OII}, \mathrm{H}_{\beta}$ and the OIII doublet are plotted in Fig. 2a. They can be classified as broad line objects (Seyfert 1/quasar) since the $\mathrm{H}_{\beta}$ line in both cases has a $F W H M \approx 2400 \mathrm{~km} \mathrm{~s}^{-1}$, broader than the forbidden lines OIII with a $F W H M \approx 1500 \mathrm{~km} \mathrm{~s}^{-1}$ (the width of the narrower lines is mainly instrumental), which implies an intrinsic broadening of $\mathrm{H}_{\beta} \approx 1900 \mathrm{~km} \mathrm{~s}^{-1}$. Seyfert galaxies and quasars are basically the same, and differ only in the proportion of light coming from the active nucleus and the host galaxy, so we do not make a distinction between these objects; the important feature is the broadening of $\mathrm{H}_{\beta}$. Figure 3 gives a zoom of Fig. 2a for the object 2 around $\mathrm{H}_{\beta}$ and OIII lines which shows better the broadening of $\mathrm{H}_{\beta}$. For the object 3 , the broadening is similar but the signal/noise ratio is smaller and perhaps not conclusive. A high contrast between the widths of the narrow and the wide lines cannot be expected because the aperture used in our slit produce a large instrumental broadening of the lines. These spectra are similar to other spectra for quasars/Seyferts 1 in other examples of anomalous redshifts (Burbidge 1995, 1997). Moreover, they are point-like objects ( $F W H M$ equal to the seeing: $\left.\approx 1.0^{\prime \prime}\right)$ which is another expected feature in this kind of objects. Other authors (Burbidge 1995, 1997; Arp et al. 2001) have also reported the detection of quasars/Seyferts 1 apparently ejected by a parent Seyfert galaxy.

If we did not trust either the argument of the broadening of $\mathrm{H}_{\beta}$ nor the argument of their being point-like objects, they would be narrow emission line extended objects. They would be HII galaxies or LINERs because $R_{23} \equiv$ $\frac{[\mathrm{OII}]+[\mathrm{OIII}]+[\mathrm{OIII}]}{\left.\mathrm{H}_{\beta}\right]}=4.2$ and 5.5 respectively for objects 2 and 3, while Seyfert 2 should have this ratio larger than 12.5 (Dessauges-Zavadsky et al. 2000). They should not be LINERs because $\frac{[\mathrm{OIIIb}]}{\mathrm{H}_{\beta}}=2.4$ and 1.0 respectively for objects 2 and 3, larger than 0.5 (Filippenko \& Terlevich 1992). Hence, they would be HII galaxies.

NGC 7603B and the filament present absorption lines, respectively with redshifts 0.058 and 0.030 (like NGC 7603). In Fig. 2b, it is shown a part of the spectra, where we can identify $\mathrm{H}_{\beta}$, OIII and MgI. The filament zones were taken as a sum of 87 columns $(0.188$ arcsec each) in the zone 1 and 173 columns in the zone 2 . Although the identification of lines for the filament is not as clear as for the emission line objects (because the signal/noise ratio is lower), we can tentatively attribute to it a redshift of 0.030 consistent with the NGC 7603 redshift. This means that we do not see a progressive change of the redshift between 0.029 and 0.057 , which would be expected if both galaxies were at the same distance and the different redshift were due to a Doppler effect of peculiar motions. This is, in some way, the least problematic of our measures. It would be ideal to have longer exposures or a larger telescope to confirm this measurement, although in any case, the most remarkable fact here is the redshift of objects 1 (NGC 7603B)-3 rather than the redshift of the filament.

NGC 7603B is a galaxy with magnitude $m_{B}=16.8$ (Sharp 1986) and we have measured magnitudes $m_{R}=21.8$ and $m_{R}=21.4$ respectively for objects 2 and 3 (Table 2). Those two objects are more prominent in blue plates than in red plates 

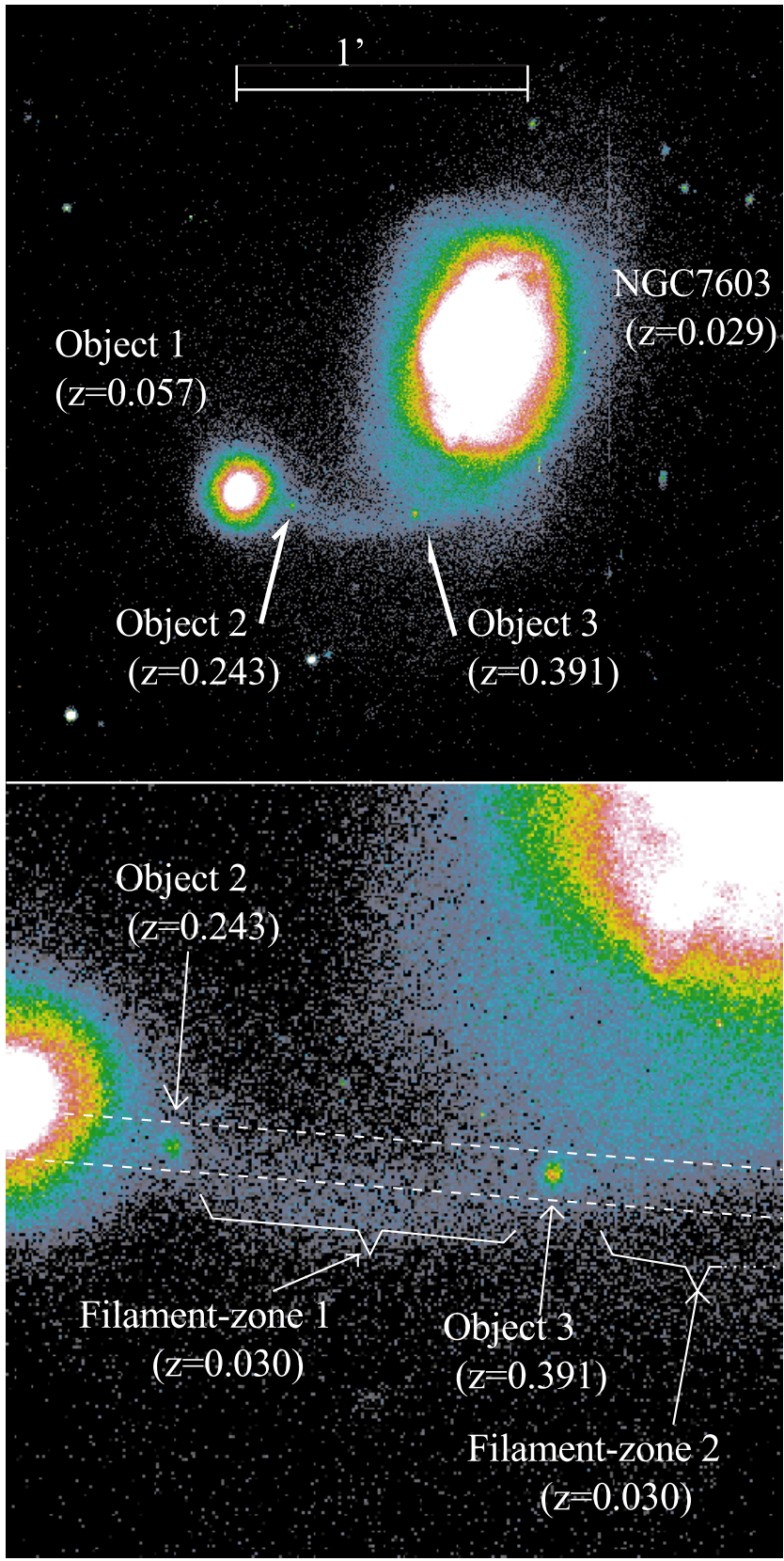

Fig. 1. a) NGC 7603 in $R$-band taken with the NOT-2.6 m. telescope (La Palma). Four objects with different redshift, plus a filament apparently connecting all of them, were observed. b) Magnification of Fig. 1a); dashed lines indicate the position of the long-slit where the spectra of the objects were taken. This figure summarizes the content of the paper: the case of a galaxy (NGC 7603) with 3 companions, all of them with different redshift. The filament between NGC 7603 and NGC 7603B (object 1) shows clearly. A knot (object 2) is centered in the line of the filament and positioned where the filament connects to NGC 7603B. The other knot (object 3) is also centered in the line of the filament, and is positioned where the filament connects with NGC 7603. The astonishing fact comes not from this image itself but from Fig. 2, which gives the redshifts of the objects. Everything points the four objects being connected among themselves, but how to explain the different redshifts? Or, in case all of them have different distances, how to explain that their projections in the sky give this extremely low probable configuration?
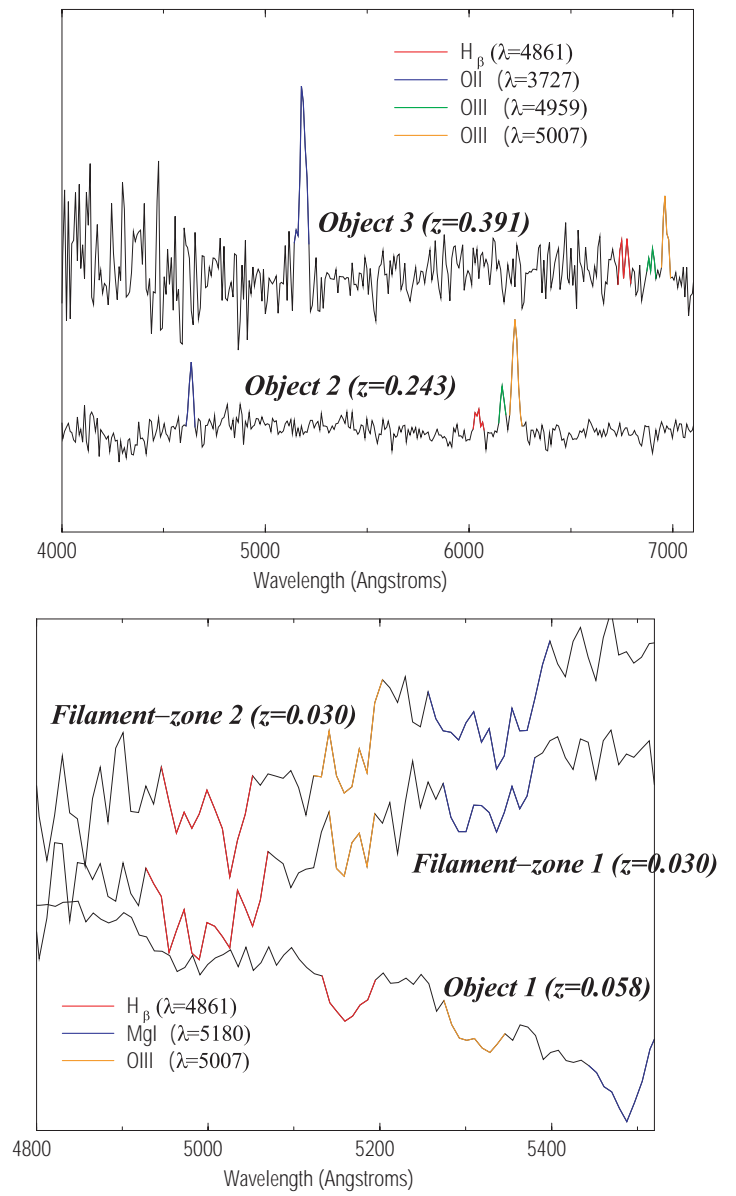

Fig. 2. a) Spectra in the range $4000-7000 \AA$ of the objects 2 and 3 (see Fig. 1). OII, OIII and $\mathrm{H}_{\beta}$ are emission lines identified in them. b) Spectra in the range $4800-5500 \AA$ of NGC 7603B and the filament in two different zones (see Fig. 1). Here we show the lines $\mathrm{H}_{\beta}$, OIII (5007 ̊), MgI in absorption. NGC 7603B has again a discordant redshift with respect NGC 7603 (already known from previous work) while the filament, with a much lower signal/noise, in both regions is presumably consistent with a redshift similar to that of NGC 7603. All the spectra were taken with the NOT-2.6 m. telescope (La Palma), continuum normalized and binned by a factor 3 for better signal/noise ratio, so the resolution in these plots is $8.9 \AA /$ pixel.

(Sharp 1986); object 2 is even visible in the blue POSSII plates (with limiting magnitude between 21.0 and 21.5), so we can be sure that the equivalent $m_{b_{j}}$ is not fainter than $\approx 21.9$, even brighter if there were some extinction through the filament. Up to these magnitudes, we have $N_{1} \sim 8 \mathrm{deg}^{-2}$ (from complete galaxy counts: Metcalfe et al. 1991); and $N_{2} \sim N_{3} \sim$ 70-350 $\mathrm{deg}^{-2}$, depending on the classification of the objects: $\sim 70$ if they were quasars/Seyfert 1 (from complete quasar counts: Boyle et al. 1991), or 5 times more if they were HII galaxies ( $10 \%$ of the emission line objects are quasars/Seyfert 1 and 50\% are HII galaxies, Ho et al. 1997; Meyer et al. 2001). That is, there should be one object like these per each square of 3-7 arcmin size (20 arcmin size for NGC 7603B); much larger than the area of the filament $\left(\sim 100 \operatorname{arcsec}^{2}\right)$. 


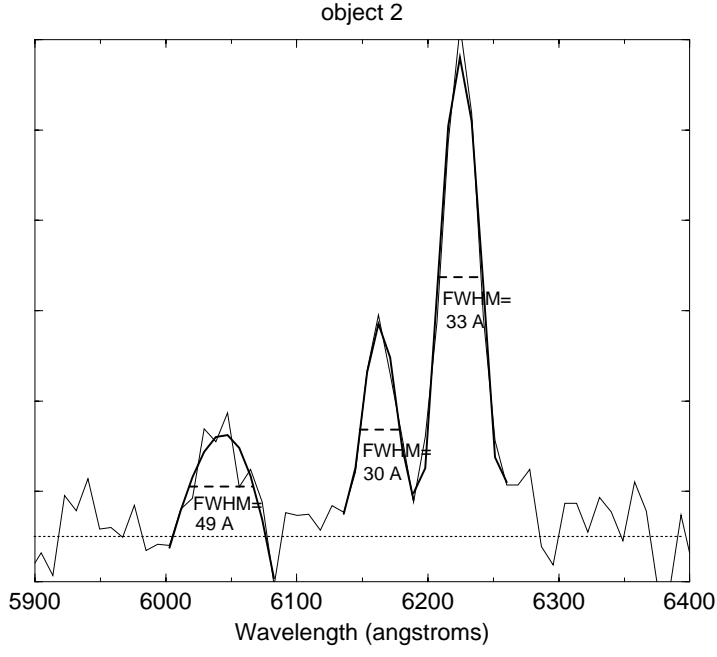

Fig. 3. Zoom of Fig. 2a for object 2. Note the excess broadening of $\mathrm{H}_{\beta}$.

\section{Discussion and conclusions}

We have clearly shown that two of the compact emission lines objects in the filament have redshifts very much greater than those of NGC7603 and its companion galaxy. Thus we have presented a very well known system with anomalous redshifts, NGC 7603, to be an apparently much more anomalous than was previously thought. There are 4 objects with very different redshifts apparently connected by a filament associated with the lower redshift galaxy. This system is at present the most spectacular case that we know among the candidates for anomalous redshift. Future studies of this system are clearly warranted.

Acknowledgements. We gratefully acknowledge the anonymous referee for helpful comments. Thanks are also given to Victor P. Debattista and Gustav Tammann (Astron. Inst. Basel) for helpful discussion about the present paper.

\section{References}

Arp, H. 1971, Astrophys. Lett., 7, 221

Arp, H. 1975, PASP, 87, 545

Arp, H., Burbidge, E. M., Chu, Y., \& Zhu, X. 2001, ApJ, 553, L11

Boyle, B. J., Jones, L. R., \& Shanks, T. A. 1991, MNRAS, 251, 482

Burbidge, E. M. 1995, A\&A, 298, L1

Burbidge, E. M. 1997, ApJ, 484, L99

Dessauges-Zavadsky, M., Pindao, M., Maeder, A., \& Kunth, D. 2000, A\&A, 355, 89

Filippenko, V. A., \& Terlevich, R. J. 1992, ApJ, 397, L79

Ho, L. C., Filippenko, A. V., \& Sargent, W. L. W. 1997, ApJ, 487, 568

Kollatschny, W., Bischoff, K., \& Dietrich, M. 2000, ApJ, 361, 901

Metcalfe, N., Shanks, T., Fong, R., \& Jones, L. R. 1991, MNRAS, 249,498

Meyer, M. J., Drinkwater, M. J., Phillips, S., \& Couch, W. J. 2001, MNRAS, 324, 343

Sharp, N. A. 1986, ApJ, 302, 245 